\title{
Hyperglycaemic presentations in type 2 diabetes
}

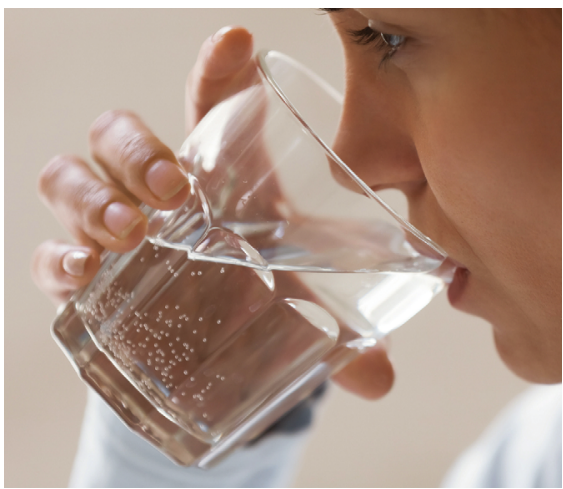

CPD

Clare Willix, Emma Griffiths, Sally Singleton

\section{Background}

Hyperosmolar hyperglycaemic state (HHS) is a syndrome that occurs in patients with type 2 diabetes mellitus (T2DM) and is comparable to diabetic ketoacidosis (DKA) seen in patients with type 1 diabetes. For a general practitioner working in a rural emergency department, recognition of HHS in a patient presenting with the triad of severe dehydration, hyperglycaemia and hyperosmolality is important to guide management and plan for disposition.

\section{Objectives}

This article reviews the hyperglycaemic states that can occur in patients with T2DM. The reasons for the biochemical derangements in both HHS and DKA are outlined, with a focus on the recognition and management of HHS.

\section{Discussion}

Knowledge of the pathophysiology that influences HHS helps understand of its clinical presentation and treatment. HHS has a high mortality rate (5-20\%), and having access to clinical guidelines from a referring hospital is useful to guide early management strategies.
DIABETES MELLITUS refers to a group of metabolic diseases characterised by hyperglycaemia, with end organ damage inflicted by the toxic effects of high plasma glucose. Symptoms of hyperglycaemia include thirst, polydipsia and polyuria. Patients with undiagnosed diabetes can present with opportunistic infections such as thrush, and hyperglycaemia can lead to blurred vision due to lens oedema. ${ }^{1}$

There are two broad pathophysiological processes that lead to a diagnosis of diabetes mellitus. ${ }^{2}$ Type 1 diabetes mellitus (T1DM) is caused by autoimmune destruction of pancreatic B cells and is defined by an absolute insulin deficiency. It usually becomes apparent in childhood or teenage years, and survival depends on exogenous insulin replacement after each carbohydrate-containing meal.

Type 2 diabetes mellitus (T2DM) is associated with insulin resistance in target tissues and pancreatic compensation with high plasma insulin levels. It typically occurs in mature-aged people. T2DM is diagnosed when the pancreas is unable to maintain sufficiently high levels of insulin to allow for the peripheral uptake of glucose, resulting in hyperglycaemia. This situation is referred to as a 'relative' insulin deficiency. ${ }^{1}$

Other rarer forms of diabetes mellitus include mature-onset diabetes in the young (MODY), late autoimmune diabetes in adults (LADA) and gestational diabetes. ${ }^{2}$ For the purposes of this article we will refer to the two main categories of diabetes mellitus, T1DM and T2DM.

The prevalence of T2DM in Australia has tripled over the past three decades, ${ }^{3}$ with an estimated one million Australian adults (5\% of the population) living with T2DM in 2014-15. ${ }^{4}$ This prevalence is based on self-reported diagnosis, and is likely an underestimate. The Australian Bureau of Statistics 2011-12 Australian Health Survey estimated that for every four adults diagnosed with T2DM, there was one who was undiagnosed. ${ }^{5}$ Although the majority of T2DM diagnoses in Australia are in people over the age of 55 years, ${ }^{3}$ there are increasing rates of young people ( $<25$ years of age) being diagnosed with T2DM, particularly in high-risk groups, including Aboriginal and Torres Strait Islander peoples. ${ }^{6}$

Some people living with poorly controlled T2DM have a remarkable capacity for physiological compensation, accommodating blood sugar levels (BSL) $>20 \mathrm{mmol} / \mathrm{L}$ without reporting symptoms. Hyperosmolar hyperglycaemic state (HHS), however, is a potentially life-threatening metabolic derangement that occurs in $\mathrm{T} 2 \mathrm{DM}$ as a result of severe hyperglycaemia. This 
syndrome was previously referred to as HHNS or HONK, an abbreviation for hyperglycemic hyperosmolar nonketotic state. It is rarer than diabetic ketoacidosis (DKA), and is reported in $<1 \%$ of hospital admissions for patients with diabetes, ${ }^{7-9}$ but has a higher mortality rate of up to $20 \%, 10$ times higher than that of patients with DKA. ${ }^{9,7}$ This increase in mortality is attributable to the older age and comorbidities (including an higher rate of venous thromboembolism) of people diagnosed with HHS. ${ }^{8}$

\section{CASE}

Brian, aged 61 years, presents to his local district hospital on a Saturday afternoon accompanied by family members. He presents with severe lethargy, weight loss (22 kg over the past three weeks), polyuria and a sore mouth. His general practitioner (GP) had told him a month ago that his fasting blood sugars indicated he was diabetic. Brian has not yet started treatment but has been focusing on lifestyle factors. In a quest to lose weight he took dietary advice from a friend and has persisted with a 'fruit only' diet, eating only grapes over a period of 72 hours. On examination he appears severely dehydrated with oral thrush, Glasgow Coma Scale 14 (eyes open to voice). His blood pressure is $112 / 93 \mathrm{mmHg}$, heart rate $120 \mathrm{bpm}$, temperature $37.8^{\circ} \mathrm{C}$ and respiratory rate 24 . Bedside point of care testing includes venous blood gas (VBG) analysis: $\mathrm{pH} 7.38$, lactate $4.4 \mathrm{mmol} / \mathrm{L}$, BSL $>27.8 \mathrm{mmol} / \mathrm{L}$ (the upper limit of testing on VBG equipment), sodium $\left(\mathrm{Na}^{+}\right) 148 \mathrm{mmol} / \mathrm{L}$, potassium $\left(\mathrm{K}^{+}\right)$ $5.1 \mathrm{mmol} / \mathrm{L}$, haemoglobin (Hb) $171 \mathrm{~g} / \mathrm{L}$ and capillary ketones $1.0 \mathrm{mmol} / \mathrm{L}$. Subsequent pathology test results include creatinine $175 \mu \mathrm{mol} / \mathrm{L}$, urea $27.4 \mathrm{mmol} / \mathrm{L}$ and eGFR $35 \mathrm{~mL} / \mathrm{min}^{2}$; glycated haemoglobin (HbA1c) is $133 \mathrm{mmol} / \mathrm{L}$ (14.3\%); BSL result from laboratory testing comes back at $48.2 \mathrm{mmol} / \mathrm{L}$. Brian's bedside troponin is negative and his white cell count is elevated. A chest X-ray shows a right-sided pneumonia.

\section{Hyperosmolar hyperglycaemic syndrome (HHS)}

HHS is the most likely diagnosis in the above case study. Risk factors for HHS include newly diagnosed T2DM, being elderly, or having reduced ability for oral fluid replacement. ${ }^{8}$ HHS is most commonly precipitated by infection, but can also arise following myocardial infarction, a cerebrovascular accident or pancreatitis. ${ }^{9}$

HHS is characterised by 1 ) severe hyperglycaemia (>30 mmol/L), which leads to an osmotic diuresis. This leads to 2) profound dehydration, hypovolaemia and 3) plasma hyperosmolality (>320 mosm $/ \mathrm{kg}$ ) with hypernatraemia. ${ }^{1,10}$ The hypernatraemia can cause an altered mental state and, in extreme cases, coma. ${ }^{11}$

High insulin levels, a feature of the insulin resistance found in T2DM, supresses lipolysis and ketogenesis. Therefore, acidosis is not a defining feature of HHS, although it can occur as part of an 'overlap' syndrome. A modified formula for calculating osmolality that can be used at the bedside is shown in Box $1 .^{10}$ The serum osmolality for the case study has been calculated as an example.

\section{Box 1. Modified formula for calculation of serum osmolality ${ }^{10}$}

Plasma osmolality $(\mathrm{mosm} / \mathrm{kg})=2(\mathrm{Na}+)+$ glucose $(\mathrm{mmol} / \mathrm{L})+$ urea $(\mathrm{mmol} / \mathrm{L})$

$=(2 \times 148)+48.2+27.4$

$=371.6 \mathrm{mosm} / \mathrm{kg}$

\section{Comparison with diabetic ketoacidosis (DKA)}

DKA, in comparison, is characterised by an absolute insulin deficiency. Similar to HHS, hyperglycaemia causes an osmotic diuresis. In the absence of insulin, glucose is unable to enter cells to allow for normal energy production. This results in peripheral lipolysis, releasing free fatty acids (FFAs) as an alternative energy substrate for cell metabolism. These FFAs are then converted into ketoacids in the liver, resulting in a metabolic acidosis (Figure 1). The biochemical criteria for diagnosing DKA include:
- hyperglycaemia (BSL >11 mmol)

- venous $\mathrm{pH}<7.3$ and/or bicarbonate $<15 \mathrm{mmol} / \mathrm{L}$

- presence of ketones in the blood (>3 mmol/L). ${ }^{1}$

As with HHS there are guidelines for management of DKA available from tertiary adult or paediatric referral centres.

\section{Differentiating between HHS and DKA}

Differentiating between HHS and DKA is important to guide management. The severe metabolic acidosis present in DKA is the distinguishing feature. A comparison of the biochemical and epidemiological features comparing the two syndromes is presented in Table 1. Clinically, Kussmaul breathing is a characteristic feature of DKA, due to respiratory compensation for metabolic acidosis; in HHS dehydration is the most obvious clinical sign. For both conditions the increase of counterregulatory hormones (glucagon, catecholamines, cortisol and growth hormone) fuel the ongoing metabolic derangements. ${ }^{9}$ Fluid and potassium replacement is a priority in both situations but insulin therapy in HHS is delayed and may cause vascular collapse if started too early (Figure 2). ${ }^{11}$

Patients with T2DM can develop ketoacidosis in some situations, presumably due to B cell dysfunction as a result of massive hyperglycaemia, and some presentations of HHS may show a mixed picture. A euglycemic ketoacidosis can also result as a rare adverse effect of the sodium-glucose co-transporter 2 inhibitors used to treat T2DM. ${ }^{1}$ In HHS, acute renal failure, precipitated by hypovolaemia, can further exacerbate hyperglycaemia. Metformin should be withheld in this situation due to the risk of lactic acidosis associated with the use of this drug. ${ }^{12}$

\section{Management of HHS}

Most patients with HHS present with profound dehydration, hypovolaemic shock or, in severe cases, coma. Principles of management follow. 


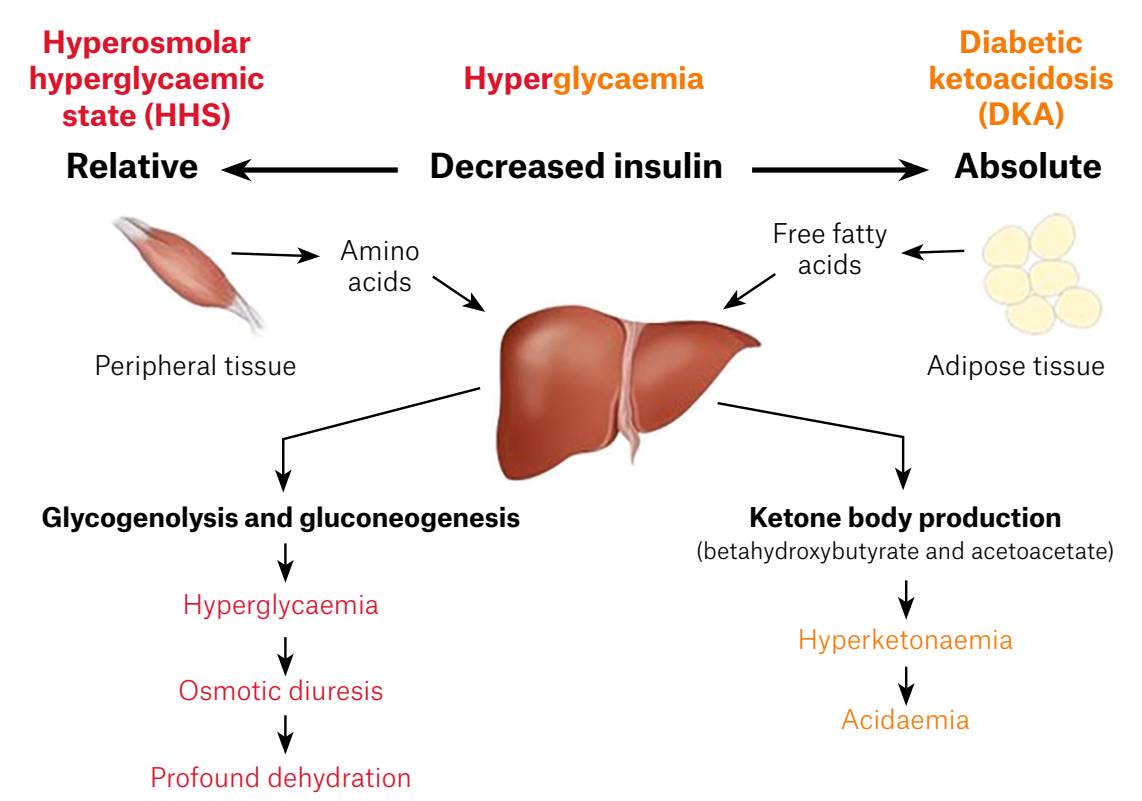

Figure 1. A comparison of the physiological changes in hyperosmolar hyperglycaemic state and diabetic ketoacidosis

Reproduced with permission from Adam Rosh.

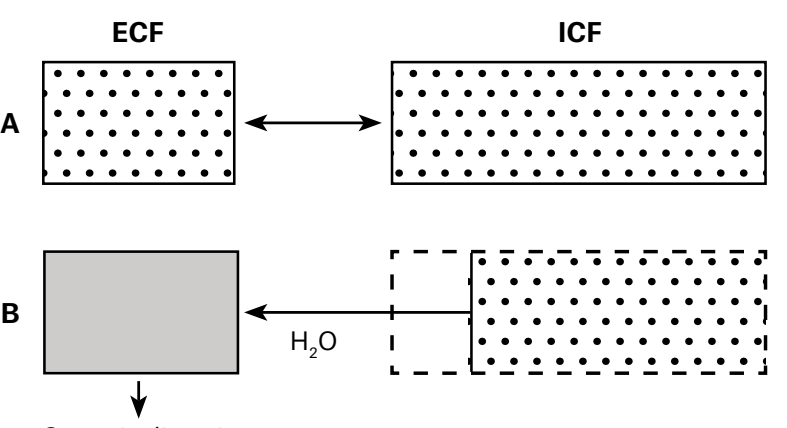

Osmotic diuresis

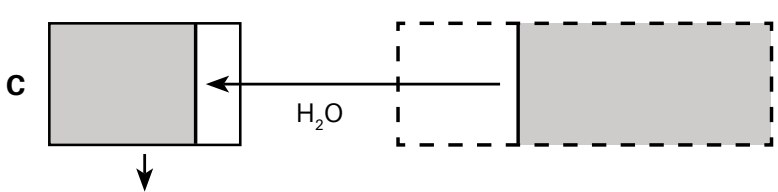

Osmotic diuresis

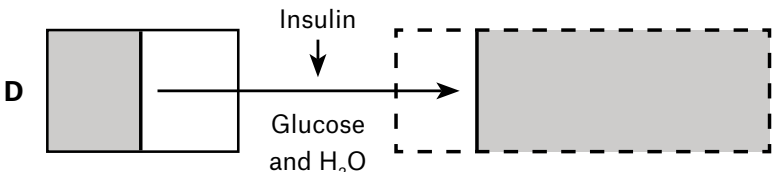

Figure 2. Fluid balance in hyperosmolar hyperglycaemic state ${ }^{11}$

A. Normoglycaemia and normal hydration; B. Early - extracellular fluid (ECF) is hyperosmolar, causing water to shift from intracellular fluid (ICF) into ECF; C. Late - continued osmotic diuresis causes dehydration, volume loss and hyperosmolality in both ICF and ECF; D. Insulin therapy without adequate fluid replacement shifts glucose and water from ECF to ICF, causing vascular collapse and hypotension.

Reproduced with permission from UK Joint British Diabetes Societies for Inpatient Care (JBDS).
- Attention to the usual resuscitation priorities should be made: securing a safe airway; ensuring adequate ventilation; followed by correction of fluid losses.

- Rehydration while maintaining electrolyte homeostasis should be the first focus of treatment. Normal saline is used, with an aim to replace 3-6 L in the first 12 hours, and 50\% of the deficit in the first 24 hours. Fluid replacement should take into consideration comorbidities such as cardiac and renal failure. Rapid reduction in serum sodium should be avoided due to the risk of cerebral oedema and central pontine myelinolysis, hence normal saline is the preferred rehydration fluid..$^{10}$ Sodium levels may increase initially; this is acceptable provided that osmolality is decreasing. The patient should be encouraged to take oral fluids as soon as safe. ${ }^{11}$ Plasma osmolality should be calculated hourly to ensure an appropriate response to therapy. An example of an appropriate rehydration schedule is shown in Table 2. The goals of treatment are to gradually correct the osmolality and electrolyte disturbances to align with the gradual onset typical of HHS. ${ }^{11}$

- As with DKA, total body potassium depletion is present in HHS despite normal plasma levels. ${ }^{9}$ Potassium should be added to the second bag of intravenous (IV) fluids unless serum levels are $>5.5 \mathrm{mmol} / \mathrm{L}$ or urine output is $<0.5 \mathrm{~mL} / \mathrm{kg}$.

- Hyperglycaemia: Rehydration alone will help to reduce BSL, and insulin infusion is only commenced if ketonemia $>1 \mathrm{mmol} / \mathrm{L}$ or if BSL reduction with IV fluids alone is $<5 \mathrm{mmol} / \mathrm{L}$ per hour. ${ }^{10}$ Figure 2 shows the fluid shifts that occur in HHS, and scenario $\mathrm{D}$ demonstrates how an over-rapid correction of blood glucose prior to rehydration can precipitate cardiovascular collapse. ${ }^{11}$ Blood glucose levels should be kept at $10-15 \mathrm{mmol} / \mathrm{L}$ for the first 24 hours, and $10 \%$ glucose commenced if blood glucose falls too quickly. Following resuscitation and return to normal fluid balance, 


\section{Table 1. Comparison of diabetic ketoacidosis and hyperosmolar hyperglycaemic state}

\begin{tabular}{|c|c|c|}
\hline & Diabetic ketoacidosis & Hyperosmolar hyperglycaemia state \\
\hline \multicolumn{3}{|l|}{ Pathophysiology } \\
\hline Insulin & Absolute deficiency & Relative deficiency \\
\hline Lipolysis/ketogenesis & +++ & $+/-$ \\
\hline Prodrome & Shorter & Longer \\
\hline \multicolumn{3}{|l|}{ Patient factors } \\
\hline Age & Younger & Older \\
\hline Diabetes type & Usually type 1 & Usually type 2 \\
\hline \multicolumn{3}{|l|}{ Biochemistry } \\
\hline Glucose & $>30$ & $>30$ \\
\hline Serum/urine ketones & Present & Absent \\
\hline \multicolumn{3}{|l|}{ Venous blood gas: } \\
\hline $\mathrm{pH}$ & $<7.3$ & $\geq 7.3$ \\
\hline Bicarbonate & $<15 \mathrm{mEq} / \mathrm{L}$ & Normal or slightly decreased \\
\hline Anion gap & Elevated +++ & Normal or slightly increased \\
\hline $\mathrm{K}+$ & Normal or increased (despite total body deficit) & Normal or increased (despite total body deficit) \\
\hline $\mathrm{Na}+$ & Hypernatraemia uncommon & Hypernatraemia common \\
\hline Serum osmolality & Variable & Hyperosmolar \\
\hline
\end{tabular}

subcutaneous insulin should be commenced. ${ }^{11}$

- Treat any underlying disease process such as infection which may have contributed to the hyperglycaemic crisis.

- Venous thromboembolism risk: The dehydration effects of HHS predispose patients to arterial and venous thromboembolism, so anticoagulation with low molecular weight heparin is recommended. ${ }^{10,11}$

- Foot care: Patients with HHS who are obtunded or uncooperative are at high risk for foot damage, so heel protection and regular foot checks are important. ${ }^{10}$

- Disposition: Ensure expeditious transfer of high-risk patients to a centre with a high dependency unit (HDU). Features of concern include plasma osmolality $>350 \mathrm{mosm} / \mathrm{kg}$, signs of shock, reduced GCS, sodium $>160 \mathrm{mmol} / \mathrm{L}$ or renal failure. ${ }^{10} \mathrm{We}$ recommend adherence to the clinical guidelines of the referring hospital to align treatment strategies, and that an early plan is made to transfer the patient to the nearest intensive care unit (ICU)/ HDU facility.

\section{Table 2. An example of an appropriate fluid regimen ${ }^{10}$}

\begin{tabular}{lll}
\hline First bag & 0.9\% sodium chloride & $1000 \mathrm{~mL}$ over 1 hour \\
\hline Second bag & $\begin{array}{l}\text { Sodium chloride } 1 \mathrm{~L} \text { with } \\
40 \mathrm{mmol} \text { potassium chloride }\end{array}$ & $1000 \mathrm{~mL}$ over next 2 hours \\
\hline Third bag & $\begin{array}{l}\text { Sodium chloride } 1 \mathrm{~L} \text { with } \\
40 \mathrm{mmol} \text { potassium chloride }\end{array}$ & $1000 \mathrm{~mL}$ over next 4 hours \\
\hline Fourth bag & $\begin{array}{l}\text { Sodium chloride } 1 \mathrm{~L} \text { with } \\
\text { 40 mmol potassium chloride }\end{array}$ & $1000 \mathrm{~mL}$ over next 4 hours \\
\hline Fifth bag & $\begin{array}{l}\text { Sodium chloride } 1 \mathrm{~L} \text { with } \\
\text { 40 mmol potassium chloride }\end{array}$ & $1000 \mathrm{~mL}$ over next 6 hours \\
\hline Reproduced with permission from Fiona Stanley Hospital & \\
\hline
\end{tabular}

\section{CASE CONTINUED}

Brian has two large bore IV cannulae inserted and IV fluids are commenced according to local HHS guidelines. ${ }^{10}$ $\mathrm{He}$ is encouraged to drink water as soon as feasible and is treated with IV antibiotics for a presumed community acquired pneumonia. He was given a $1 \mathrm{mg} / \mathrm{kg}$ dose of subcutaneous clexane and his heels are protected. Brian was transferred by road to a regional hospital and spends two days in the
HDU. After transfer to the medical ward he receives education about diabetes and is discharged home five days later on twice-daily subcutaneous insulin.

\section{Key points}

- Patients with T2DM often present with non-specific symptoms, but can rarely present as a hyperglycaemic emergency. 
- The triad of severe dehydration, hyperglycaemia and osmolality >320 msom/L, along with normal ketones and normal bicarbonate, points to a diagnosis of HHS. This is in contrast to the acidosis and dehydration seen in DKA. An explanation for the variation in the two presentations relates to the availability or absence of endogenous insulin.

- Management priorities for patients with HHS include recognition of the underlying cause, gradual reversal of electrolyte, glucose and osmolality abnormalities via judicious fluid replacement, and planning for transfer to a centre with ICU/HDU facilities.

- Treatment guidelines from referral hospitals should be readily available in rural emergency departments to guide treatment and avoid complications prior to transfer.

\section{Authors}

Clare Willix MBBS, FRACGP, FACRRM, Clinical Senior Lecturer, Rural Clinical School of Western Australia,Bunbury,WA.clare.willix@rcswa.edu.au
Emma Griffiths MBBS, MPHTM, FRACGP, FARGP, FAFPHM, Clinical Senior Lecturer, Rural Clinical School of Western Australia, Broome, WA

Sally Singleton MBBS, BMedSc, MPHTM, FAFPHM FRACGP, FACRRM, Clinical Senior Lecturer, Rural Clinical School of Western Australia, Broome, WA

Competing interests: None.

Funding: None.

Provenance and peer review: Commissioned, externally peer reviewed.

\section{References}

1. Royal Australian College of General Practioners. General practice management of type 2 diabetes 2016-2018. East Melbourne, Vic: RACGP, 2016. Available at www.racgp.org.au/clinical-resources/ clinical-guidelines/key-racgp-guidelines/view-allracgp-guidelines/management-of-type-2-diabetes [Accessed 20 March 2019].

2. American Diabetes Association. Diagnosis and classification of diabetes mellitus. Diabetes Care 2014;37 Suppl 1:S81-90. doi: 10.2337/dc14-S081.

3. Australian Institute of Health and Welfare. Diabetes. Canberra: AlHW, 2019. Available at www.aihw.gov.au/reports-data/health-conditionsdisability-deaths/diabetes/overview [Accessed 20 March 2019].

4. Australian Bureau of Statistics. National Health Survey: First Results, 2014-15. Diabetes mellitus. Cat. no. 4364.0.55.001. Canberra: ABS, 2015 Available at www.abs.gov.au/ausstats/abs@.nsf/ Lookup/by\%20Subject/4364.0.55.001 201415 Main\%20Features Diabetes\%20mellitus 12 [Accessed 3 April 2019]

5. Australian Bureau of Statistics. National Health Survey: Updated results, 20112012. Cat. no. 4364.0.55.003. Canberra: ABS, 2013. Available at www.abs.gov.
au/ausstats/abs@.nsf/Lookup/1A8F3 DE217DE1057CA257B82001792F4? opendocument [Accessed 9 April 2019].

6. Australian Institute of Health and Welfare. Type 2 diabetes in Australia's children and young people: A working paper. Canberra: AlHW, 2014. Available at www.aihw.gov.au/reports/diabetes/type-2diabetes-children-young-people/notes [Accessed 3 April 2019].

7. Pasquel FJ, Umpierrez GE. Hyperosmolar hyperglycaemic state: A historic review of the clinica presentation, diagnosis and treatment. Diabetes Care 2014;37(11):3124-31. doi: 10.2337/dc14-0984.

8. Avichal D. Hyperosmolar hyperglycemic state. Medscape, 2019. Available at https://emedicine. medscape.com/article/1914705-overview. [Accessed 20 March 2019].

9. Kitabchi AE, Umpierrez GE, Miles JM, Fisher JN. Hyperglycaemic crises in adult patients with diabetes. Diabetes Care 2009;32(7):1335-43. doi: 10.2337/dc09-9032.

10. Fiona Stanley Hospital. Clinical guidelines: Adult hyperosmolar hyperglyacaemic state - Ref:FSHEND-GUI-0003. Murdoch, WA: Fiona Stanley Hospital, 2014.

11. Joint British Diabetes Societies Inpatient Care Group. The management of the hyperosmolar hyperglycaemic state (HHS) in adults with diabetes. UK: National Health Service, 2012. Available at www.diabetologists-abcd.org.uk/ JBDS/JBDS_IP_HHS_Adults.pdf [Accessed 9 April 2019].

12. BMJ best practice guidelines. Hyperosmolar hyperglycaemic state. London: BMJ Publishing Group, 2017. Available at https://bestpractice.bmj. com/topics/en-gb/1011 [Accessed 3 April 2019]. 